\title{
Analysis of commonly expressed genes between first trimester fetal heart and placenta cell types in the context of congenital heart disease
}

Rebecca L. Wilson ${ }^{1,2 *}$, Victor Yuan ${ }^{3,4}$, Jennifer Courtney ${ }^{5}$, Alyssa Tipler ${ }^{1,2}$, James Cnota ${ }^{6}$, Helen N. Jones ${ }^{1,2}$

${ }^{1}$ Department of Physiology and Functional Genomics, University of Florida College of Medicine, Gainesville, Florida USA 32610

${ }^{2}$ Center for Research in Perinatal Outcomes, University of Florida, Gainesville, Florida USA 32610

${ }^{3} \mathrm{BC}$ Children's Hospital Research Institute, 950 W 28th Ave, Vancouver, V6H 3N1, Canada

${ }^{4}$ Department of Medical Genetics, University of British Columbia, 4500 Oak St, Vancouver, V6H

$3 \mathrm{~N} 1$, Canada

${ }^{5}$ Center for Fetal and Placental Research, Cincinnati Children's Hospital Medical Center, Cincinnati, Ohio USA 45229

${ }^{6}$ Heart Institute, Cincinnati Children's Hospital Medical Center, Cincinnati, Ohio USA 45229

* Corresponding author

Rebecca Wilson

Center for Research in Perinatal Outcomes

University of Florida

Gainesville, Florida 32610

Phone: (352) 846-1503

Email: rebecca.wilson@ufl.edu

Congenital heart disease (CHD) is often associated with fetal growth abnormalities. During the first trimester of pregnancy, the heart and placenta develop concurrently, and share key developmental pathways. Hence, it is hypothesized that defective morphogenesis of either organ is synergistically linked. However, many studies determined to understand the mechanisms behind CHD overlook the contribution of the placenta. In this study, we aimed to identify commonly expressed genes between first trimester heart and placenta cells using two publicly available single cell sequencing databases. Using a systematic computational approach, we identified 328 commonly expressed genes between heart and placenta endothelial cells and enrichment in pathways including Vasculature Development (GO:0001944, FDR 2.90E-30), and Angiogenesis (GO:0001525, FDR 1.18E-27). We also found, in comparison with fetal heart endothelial cells, 197 commonly expressed genes with placenta extravillous trophoblasts, 128 with cytotrophoblasts and 80 with syncytiotrophoblasts, and included genes such as FLT1, GATA2, ENG and CDH5. Finally, comparison of first trimester cardiomyocytes and placenta cytotrophoblasts revealed 53 commonly expressed genes and enrichment in biological processes integral to cellular function including Cellular Respiration (GO:0045333; FDR 5.05E-08), lon Transport (GO:0006811; FDR 2.08E-02), and Oxidation-Reduction Process (GO:0055114; FDR 1.58E-07). Overall, our results identify specific genes and cellular pathways common between first trimester fetal heart and placenta cells which if disrupted may concurrently contribute to the developmental perturbations resulting in CHD. 
Congenital heart disease, first trimester, heart, placenta, commonly expressed genes

\section{Introduction}

Congenital heart disease (CHD) is the most common birth defect ${ }^{1}$. Affecting approximately $1 \%$ of live births, it is the leading cause of infant mortality related to birth defects ${ }^{2}$, and is often the result of perturbations in normal programming of cardiac development. In approximately one third of babies, the CHD is classified as severe and requires intervention in the first year of life ${ }^{3}$. Survival of such interventions is hindered by the fact that there is a higher incidence of fetal growth restriction (FGR) and preterm birth in CHD pregnancies. Such associations suggest that the perturbations to cardiac programming are also affecting placental development, however, the etiology of fetal growth abnormalities in CHD is largely unknown.

In utero the placenta and heart develop concurrently ${ }^{4-8}$. Placenta and heart development also share key developmental pathways; hence it is reasonable to assume that deleterious changes in gene expression during first trimester development of one are likely to perturb morphogenesis of the other ${ }^{9}$. The heart is the first organ to develop within the fetus with cells destined to become the mature heart originating from the mesoderm ${ }^{10}$. The asymmetrical nature of heart development at both the organ and tissue level contribute to the complex nature of the molecular events which occur in the first trimester. At the same time as the early heart is developing, vascular development of the placenta is also occurring ${ }^{11}$. Arising from the trophectoderm and extra-embryonic mesoderm, trophoblastic structures branch and give rise to the primary and secondary placental villi. Differentiation of the mesenchymal cells inside the villi eventually result in the first hemangiogenic precursor cells and ultimately develop into the placental vasculature.

In both heart and placental development, the cellular and signaling events that occur during the first trimester will ultimately determine the fate of the developing heart and placental vasculature. Numerous animal models have shown that disrupted expression of genes associated with common vasculogenesis and angiogenesis pathways result in both placental and cardiac defects ${ }^{12}$. In addition to molecular signals which modulate heart development in the first trimester, mechanical forces are also required. For example, there is evidence which suggests that ventricular wall expansion in the heart switches from embryonic to placental control after the onset of maternal blood flow into the placenta ${ }^{13}$. Thus, there is the potential in pregnancies complicated by diseases such as preeclampsia or FGR, where there is inadequate or changed placental blood flow, that a synergistic effect with placental insufficiency exacerbates the developmental defect in the heart ${ }^{9}$.

The parallel development of the placenta and heart in utero has led to the hypothesis that defective morphogenesis of either organ is synergistically linked. Indeed, there are several mutant mouse models which exhibit both defective heart and placenta development ${ }^{9}$. In some cases, the heart defects were shown to be secondary to defective placental development, possibly as a consequence of insufficient placental blood flow affecting early embryonic cardiac function ${ }^{14}$. However, despite the reoccurring link between heart and placental defects, studies focused on CHDs rarely acknowledge the potential contribution of the placenta. It is estimated that over 300 genes collectively contribute to CHDs in humans ${ }^{15}$, and studies using conditional knockout animal models have confirmed that disrupting many of these genes leads to cardiac defects ${ }^{16}$. In these 
studies, conditional knockout occurred in cardiac tissue only, however, many of the genes are known to also be expressed in the placenta. Therefore, there is a heightened need to identify and better understand common gene expression profiles between the heart and placenta, particularly in the first trimester, in order to improve the understanding of the mechanisms by which CHDs occur.

Advances in single-cell sequencing techniques are now allowing for greater in-depth analyses of how a given gene is expressed in different organs like the placenta and heart. In this study, we utilize two publicly available databases analyzing gene expression in first trimester human placenta ${ }^{17}$ and fetal heart cells ${ }^{18}$. Using a systematic, computational approach, we aimed to identify commonly expressed genes between first trimester placenta endothelial cells (ECs), extravillous trophoblasts (EVTs), cytotrophoblasts (CTBs) and syncytiotrophoblasts (STBs), and first trimester fetal heart ECs and cardiomyocytes. Our results identify specific genes and cellular pathways common between placental and fetal heart cells which if disrupted may concurrently contribute to the developmental perturbations resulting in CHD.

\section{Results and Discussion}

Using publicly available databases ${ }^{17,18}$, we compared single-cell gene expression profiles from first trimester human embryonic heart ECs and cardiomyocytes with first trimester human placental ECs, EVTs, CTBs and STBs. Principal component analysis based on global gene expression showed clustering of fetal heart and placenta ECs, whilst EVTs, CTBs, and STBs also clustered together (Figure 1). Compared to heart ECs, there was 328 commonly expressed genes with placental EC, 197 with EVTs, 128 with CTBs and 80 with STBs (Figure 1). For heart cardiomyocytes, there was 16 commonly expressed genes with placental endothelial cells, 36 genes in common with EVTs, 53 genes with CTBs and 22 commonly expressed genes with STBs (Figure 1). Full details of commonly expressed genes and pathway enrichment analysis can be found in Supplemental Material.

\section{Commonly expressed genes between first trimester placental and fetal heart endothelial cells}

As expected, heart and placental endothelial cells had the most commonly expressed genes. Furthermore, functional enrichment analysis using ToppGene - ToppFun revealed enrichment of genes associated with biological functions including Vasculature Development (GO:0001944, FDR 2.90E-30), Angiogenesis (GO:0001525, FDR 1.18E-27), Heart Development (GO:0007507, FDR 9.15E-12) and In utero Embryonic Development (GO:0001701 FDR 6.84E-07)(Figure 2a), reflecting the role of endothelial cells in both the placenta and heart. Early in heart development, the primitive heart consists of 2 cardiac progenitor cell layers: endocardial endothelial cells and cardiomyocytes ${ }^{19}$. Originating from the rostrolateral mesoderm, it is the endocardial endothelial cells which differentiate and give rise to the other cell types of the heart including cells in the cardiac valves and chambers ${ }^{20}$. Similarly in the placenta, precursor endothelial cells are derived from the mesoderm ${ }^{21}$. Vasculogenesis begins within the first 18-20 days after conception ${ }^{22}$. Precursor endothelial cells form vessels beneath the trophoblastic epithelium through a combination of cell replication and stromal cell recruitment, with villous circulation formed by 6 weeks post-conception. As pregnancy progresses, there is expansion of the fetal capillary bed 

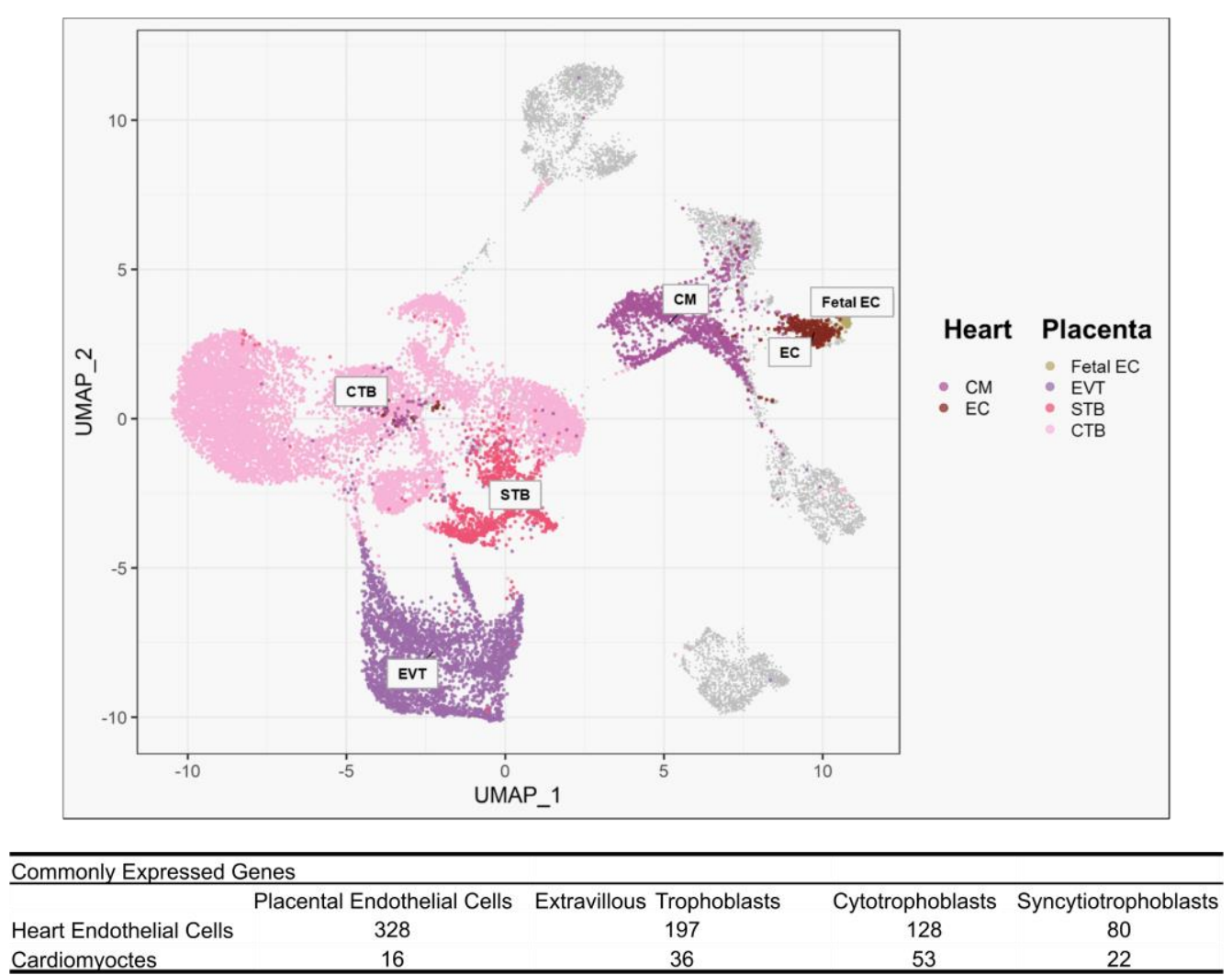

Figure 1. Principal component analysis of global gene expression in first trimester heart and placenta cell types and number of commonly expressed genes between heart endothelial cells (EC) or cardiomyocytes (CM) and placenta (fetal) EC, extravillous trophoblasts (EVT), syncytiotrophoblasts (STB) and cytotrophoblasts (CTB).

via both branching and non-branching angiogenesis in order to fully support rapid growth of the fetus in late pregnancy ${ }^{7}$.

We also assessed commonly expressed genes between heart endothelial cells and placental endothelial cells for enrichment in mouse phenotypes. This revealed enrichment for phenotypes such as Abnormal Heart Development (MP:0000267 FDR 7.27E-05) and Abnormal Placental Vasculature (MP:0003231 FDR 4.92E-04). Moreover, there was enrichment in numerous mouse phenotypes associated with abnormal placental development and function including Embryonic Growth Retardation (MP:0003984 FDR 6.25E-06), Lethality throughout Fetal Growth and Development (MP:0006208 FDR 1.25E-08) and Abnormal Visceral Yolk Sac Morphology (MP:0001718 FDR 2.96E-10). Comparison of the 26 genes associated with abnormal heart development and abnormal placental vasculature showed 8 genes similar to both phenotypes including notch receptor 1 (NOTCH1) and its receptor ligand DLL4 (Figure 2b). Notch signaling is highly conserved and provides a means by which cells can influence neighboring cells through receptor-ligand binding ${ }^{23}$. In the heart, Notch signaling has been shown to regulate cardiac cell fate and orchestrate cardiac chamber and valve morphogenesis ${ }^{24}$. Additionally, there are several Notch pathway genes in which mutations have been implicated in CHDs including NOTCH1, NOTCH2, DLL4, JAG1 and MAML2 ${ }^{15}$. Mutations in NOTCH1 are associated with numerous CHDs ranging from issues with development of the bicuspid aortic valve to HLHS ${ }^{25}$. 
In terms of the placenta, Notch $1^{-/}$knockout mice are embryonically lethal at gestational day 11.5 26. Analysis of the placenta has shown that whilst fusion of the allantois with the chorionic plate occurs, fetal blood vessels do not form within the labyrinthine region of the placenta ${ }^{27}$. This phenotype has also been reported in a mouse model where Notch1 was conditionally knockout only in the endothelium ${ }^{28}$ and as such, indicates a major role for Notch1 in placental development.

From a translational perspective, there was enrichment in genes commonly expressed between placental and heart ECs for Human Congenital Abnormalities (C0000768 FDR 5.43E06). The 42 genes included a specific cluster of genes, including NOTCH1, HIF1A, KDR, and NOS3, in which there are known protein-protein interactions (Figure 2c). NOS3, or endothelial nitric oxide synthase is one of three nitric oxide synthase isoforms and is crucial in the regulation of vascular integrity and homeostasis. In pregnancy, the importance of NOS3, particularly towards placenta angiogenesis and vascular development is well established ${ }^{29}$. Nos3 knockout mice are characterized with fetal growth restriction during pregnancy and placental dysfunction ${ }^{30,31}$. Additionally, genetic variants in NOS3 gene have been associated with increased risk of the pregnancy disease preeclampsia ${ }^{32}$. In terms of cardiac development, NOS 3 is known to play an

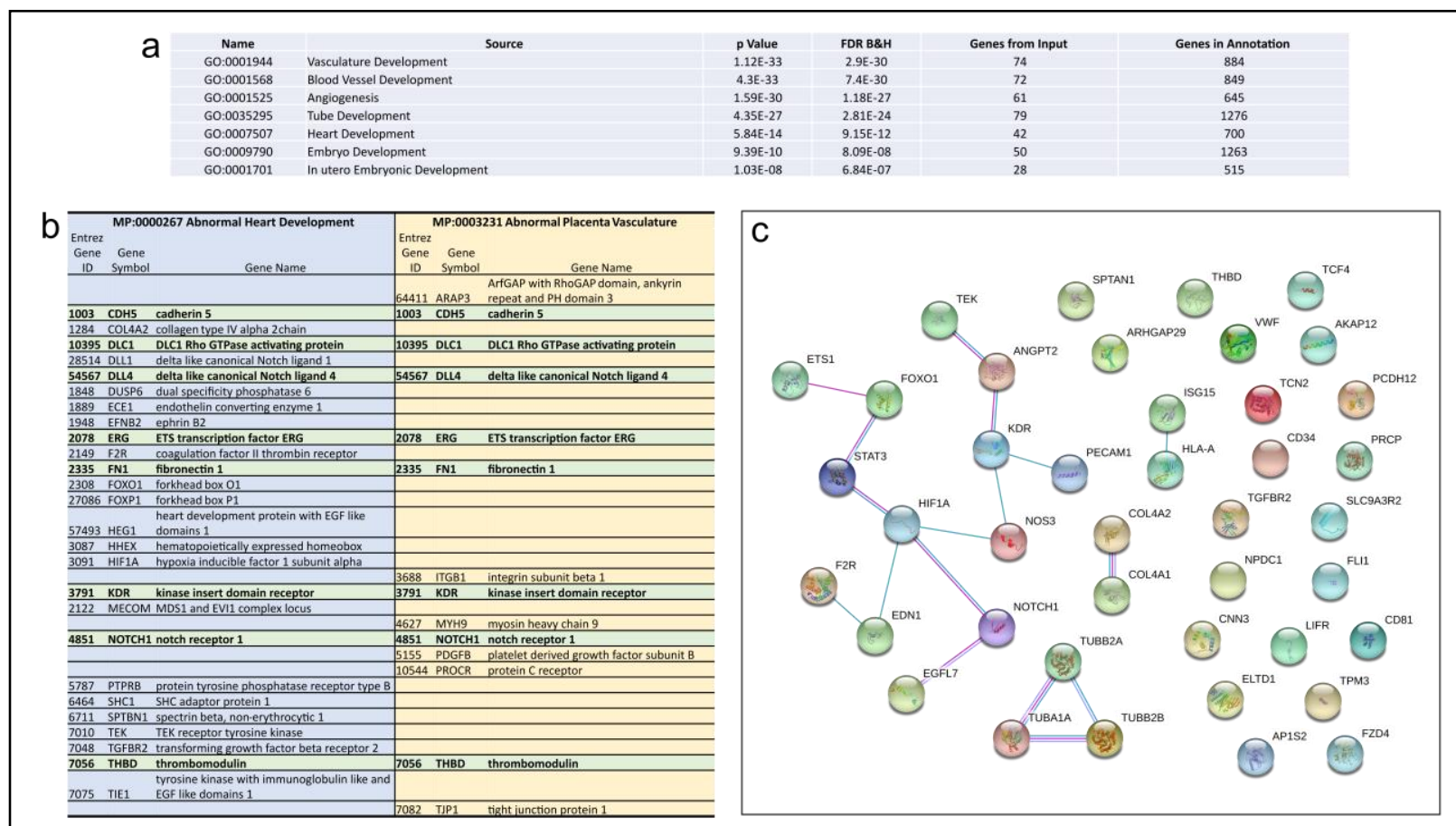

Figure 2. Comparison of commonly expressed genes between first trimester placenta and heart endothelial cells. a. Enrichment analysis of GoBiological processes of the commonly expressed genes. b. Comparison of commonly expressed genes between placenta and heart endothelial cells associated with abnormal heart development and placenta vasculature in mouse phenotypes. c. Protein-protein interactions of 42 commonly expressed genes between placenta and heart endothelial cells associated with human congenital diseases.

important role ${ }^{33,34}$ and polymorphisms in NOS3 have been shown to be associated with increased risk of sporadic CHD, and more specifically a $62 \%$ increased risk of perimembranous ventricular septal defects ${ }^{35}$. 


\section{Commonly expressed genes between first trimester heart endothelial cells and first trimester placenta trophoblasts}

Early placental development and function is determined by the various groups of trophoblasts; disrupted cellular signaling events within these trophoblast cells can lead to defective placentation and the development of obstetric diseases. There were 197 commonly expressed genes between fetal heart ECs and placental EVTs and included enrichment in biological functions such as, Vascular Development (GO:0001944, FDR 2.03E-05), Angiogenesis (GO:0001525, FDR 1.83E05) as well as, Cell Migration (GO:0016470 FDR1.21E-05), Cell Adhesion (GO:0007155 FDR 2.26E-05) and Extracellular Structure Organization (GO:0043062 FDR 1.25E-07)(Figure 3a). Enrichment in such pathways is unsurprising given the roles of both heart ECs and EVTs in their respective organs. Coinciding with the time in pregnancy in which the early heart patterning is occurring, the placental EVTs invade the maternal uterine decidua, transforming the uterine spiral arteries in preparation for the onset of placental blood flow ${ }^{36}$. Numerous obstetric diseases including FGR and preeclampsia are characterized by having abhorrent maternal spiral artery transformation, most likely due to inappropriate functioning of the EVTs ${ }^{37}$. Large human cohort studies have also confirmed strong positive associations between congenital heart defects and obstetric diseases like preeclampsia, preterm birth and FGR ${ }^{38-42}$ further strengthening the hypothesis that the underlying pathophysiology of CHDs and placental-related pregnancy complications originate from similar biological insults.

In terms of mouse phenotypes, there was enrichment in the commonly expressed genes between fetal heart ECs and EVTs for Abnormal Vascular Development (M:0000259 FDR 3.27E05) and Abnormal Cardiovascular System Physiology (M:0001544 FDR 9.66E-05) and included genes such as MMP2, FN1, ENG and EPAS1. Interestingly, there was also enrichment for genes associated with Abnormal Hormone Levels (MP:0003953 FDR 1.55E-02) and included SOX4, GATA2, and GATA3 which are known to interact at a protein level (Figure $3 \mathrm{~b}$ ), as well as Abnormal Inflammatory Response (MP:0001845 FDR1.55E-05) including genes JAK1, IL1R1, and IFNGR1 (Figure 3c). The role of the immune system in placental development and function is well established with the invading EVTs communicating with the resident uterine immune cells to invade and transform the uterus appropriately ${ }^{43}$. Improper control of EVT invasion is a hallmark characteristic of placenta accreta disorder, in which EVT invasion extends beyond the maternal decidua and in severe cases, into other organs like the bowel or bladder and more is being understood about the role of immune cells in allowing such over invasion to occur ${ }^{44}$. Both overand under-invasion of the EVTs results in significant changes to placental hemodynamics and the flow of maternal blood into the placenta. It is known that early cardiac development is dependent on both genetic and environmental factors, and hemodynamic forces associated with blood flow play an important role ${ }^{45,46}$. Experimentally induced alterations in hemodynamics of the umbilical vein and umbilical artery have been shown to trigger detrimental growth and remodeling cascades eventuating in major cardiac defects ${ }^{47}$. Such findings support the idea that impaired maternal blood flow to the placenta, as well as genetic factors, can have a significant effect on early embryonic cardiac development and help explain why there is a strong association between placental-related pregnancy complications and CHDs.

Whilst the functional similarities between fetal heart ECs and placenta CTBs and STBs are not as well defined as between fetal heart ECs and placenta ECs and EVTs, there was still a large number of commonly expressed genes. Enrichment analysis of the 128 commonly 
expressed genes between fetal heart ECs and placental CTBs revealed biological processes involved in cellular function, such as, Exocytosis (GO:0006887 FDR 1.71E-02), Glycoprotein Metabolic Process (GO:0009100 FDR 8.07E-03), Membrane Fusion (GO:0061025 FDR 2.33E02), Response to Endogenous Stimuli (GO:0009719 FDR 1.14E-02) and Vesicle Organization (GO:0016050 FDR 2.33E-02) as opposed to vasculature development (Figure 4a). On the other hand, commonly expressed genes between fetal heart ECs and STBs were enriched for biological processes including Angiogenesis (GO:0001525 FDR 3.76E-05), Heart Morphogenesis (GO:0003007 FDR 1.31E-02) and Tube Morphogenesis (GO:0035239 FDR 2.46E-05), as well as functions like Secretion (GO:0046903 FDR 5.90E-04) and Organelle Fusion (GO:0048284 FDR 2.00E-04) more typically associated with STB function (Figure 4b). There was also enrichment in mouse phenotypes associated with Embryonic Lethality During Organogenesis, complete penetrance (MP:0011098 FDR 5.50E-03) and Abnormal Heart Morphology (MP:0000266 FDR

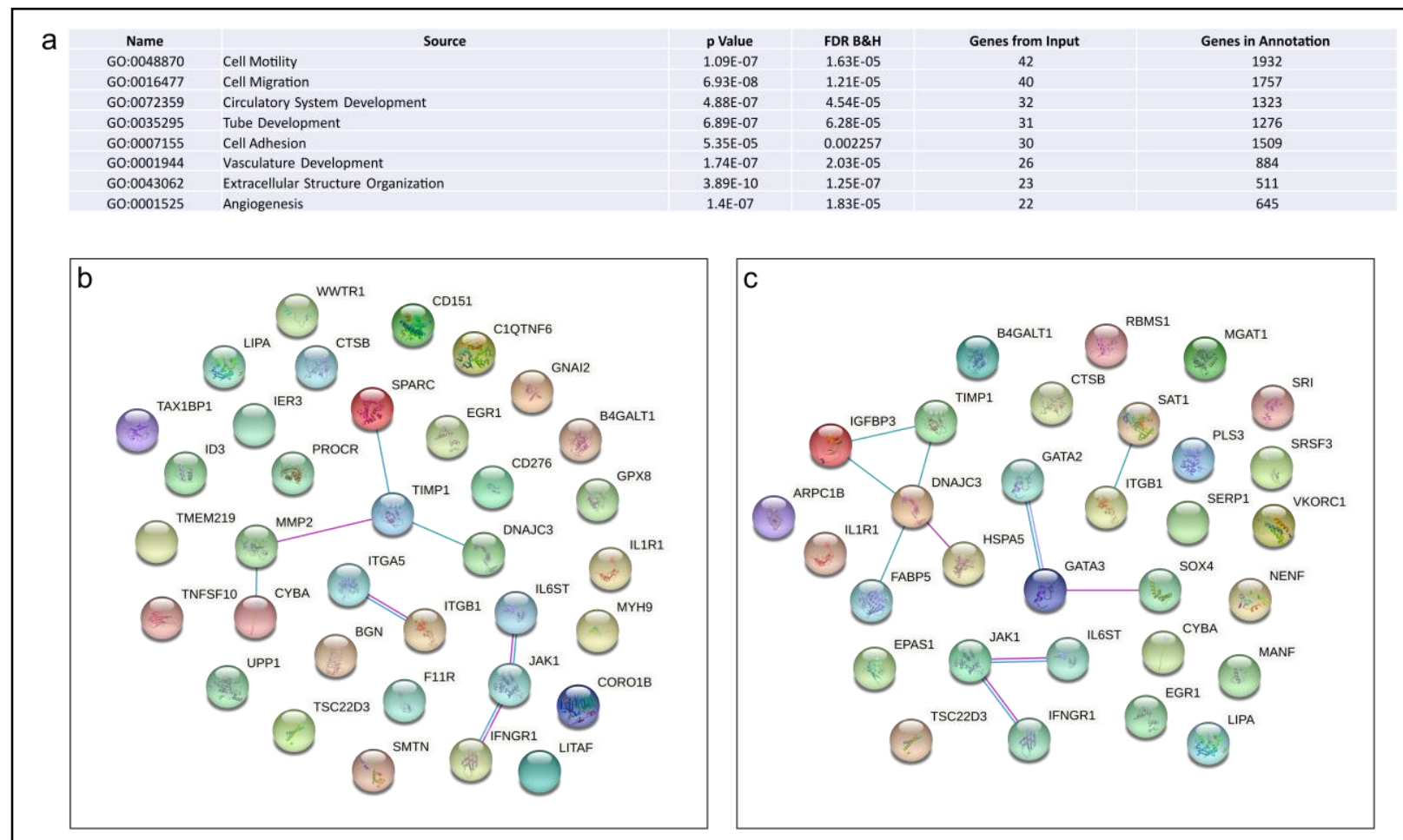

Figure 3. Comparison of commonly expressed genes between fetal heart endothelial cells and placenta extravillous trophoblast cells. a. GoBiological processes enrichment analysis of the commonly expressed genes. b. Protein-protein interactions of commonly expressed genes associated with abnormal hormone levels. c. Protein-protein interactions of commonly expressed genes associated with abnormal inflammation response.

1.14E-02) and included genes such as FLT1, GATA2, ENG and CDH5 which were common to both phenotypes (Figure 4c). Analysis of protein-protein interactions between the 80 commonly expressed genes revealed a unique cluster of 6 genes hypothesized to be involved in the innate immune response (Figure $4 d$ ). 


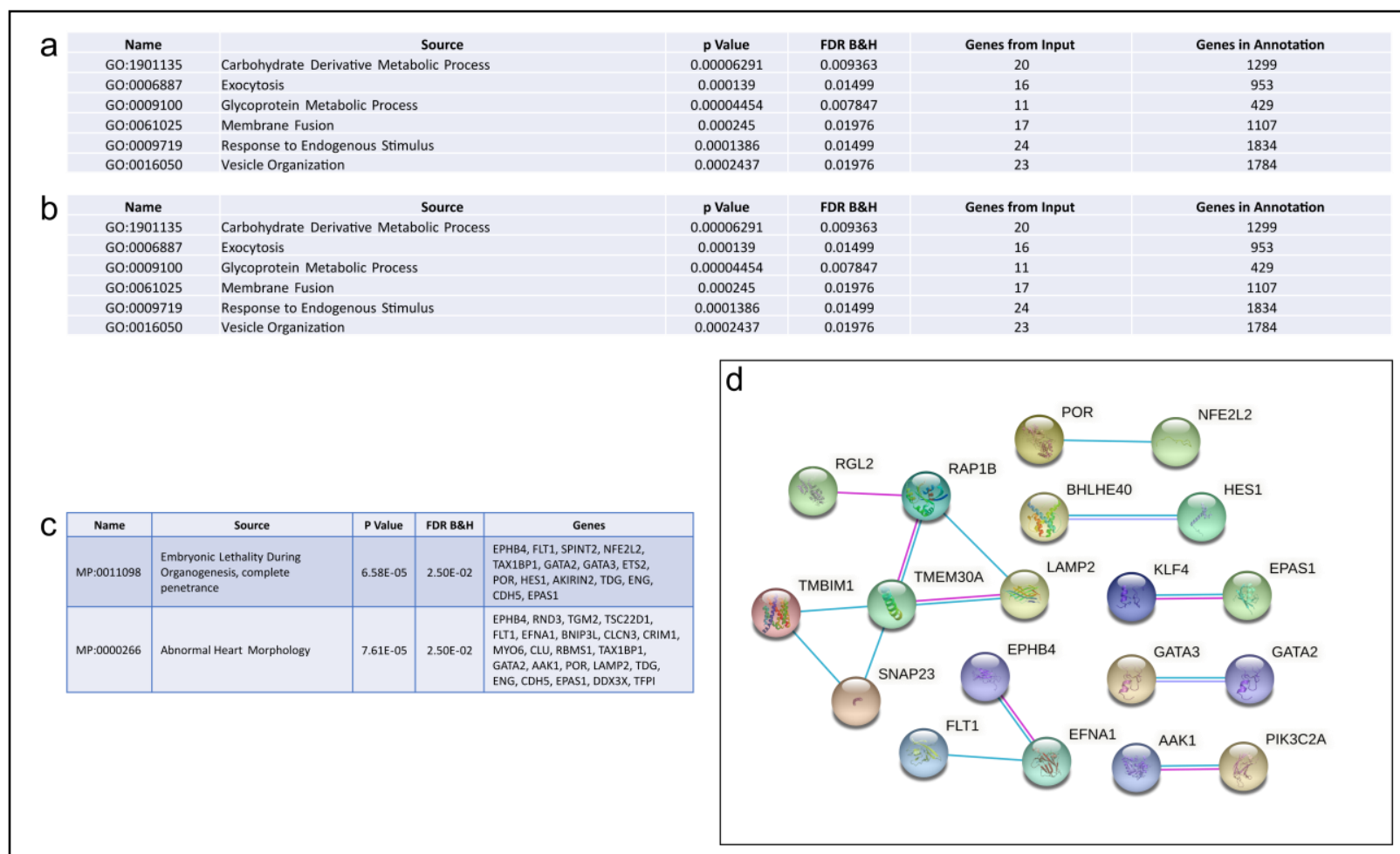

Figure 4. Comparison of commonly expressed genes between fetal heart endothelial cells and placenta cytotrophoblasts and syncytiotrophoblasts. a. GoBiological processes enrichment analysis of commonly expressed genes between heart endothelial cells and placenta cytotrophoblasts. b. GoBiological processes enrichment analysis of commonly expressed genes between heart endothelial cells and placenta syncytiotrophoblasts. c. Enrichment analysis of commonly expressed genes between heart endothelial cells and syncytiotrophoblasts in mouse phenotypes. d. Protein-protein interaction of the 80 commonly expressed genes between first trimester fetal heart endothelial cells and placenta syncytiotrophoblasts.

\section{Commonly expressed genes between first trimester cardiomyocytes and first trimester placenta trophoblasts}

Despite overall gene expression profiles clustering first trimester fetal cardiomyocytes close to first trimester placenta ECs, there were only 16 commonly expressed genes between the two cell types and included the glucose transporter SLC2A1, AK1, CAV1, and BEX1. Similarly, there were very few commonly expressed genes between fetal heart cardiomyocytes and placenta EVTs and STBs: see supplemental material for detailed list of commonly expressed genes. However, comparison of fetal heart cardiomyocytes and placenta CTBs; both cell types are progenitor cells in their respective organs, revealed 53 commonly expressed genes including BMP7, SLC38A1, GJA1, and DSP (Figure 5a). These 53 genes were enriched for biological processes integral to cellular function including Cellular Respiration (GO:0045333; FDR 5.05E-08), Ion Transport (GO:0006811; FDR 2.08E-02), and Oxidation-Reduction Process (GO:0055114; FDR 1.58E07)(Figure 5b). In the heart, cardiomyocytes are responsible for driving heart contraction, maturing from fetal cardiomyocytes to adult cardiomyocytes in order to sustain cycles of contraction and relaxation ${ }^{48}$. Cardiomyocyte regeneration occurs naturally through proliferation 
of existing cardiomyocytes ${ }^{49}$, although this proliferative capacity only exists during fetal development and is quickly lost after birth ${ }^{50}$. In the placenta, CTBs undergo asymmetrical division where by one daughter cell re-populates the progenitor pool whilst the other differentiates and fuses with the overlying STB layer ${ }^{51}$. For both cardiomyocytes and CTBs, maladaptive responses in differentiation and function are characteristic of pathological conditions including hypertension, myocardial infarction, and cardiac fibrosis in the heart ${ }^{52}$, and preeclampsia and FGR in the placenta ${ }^{53}$.

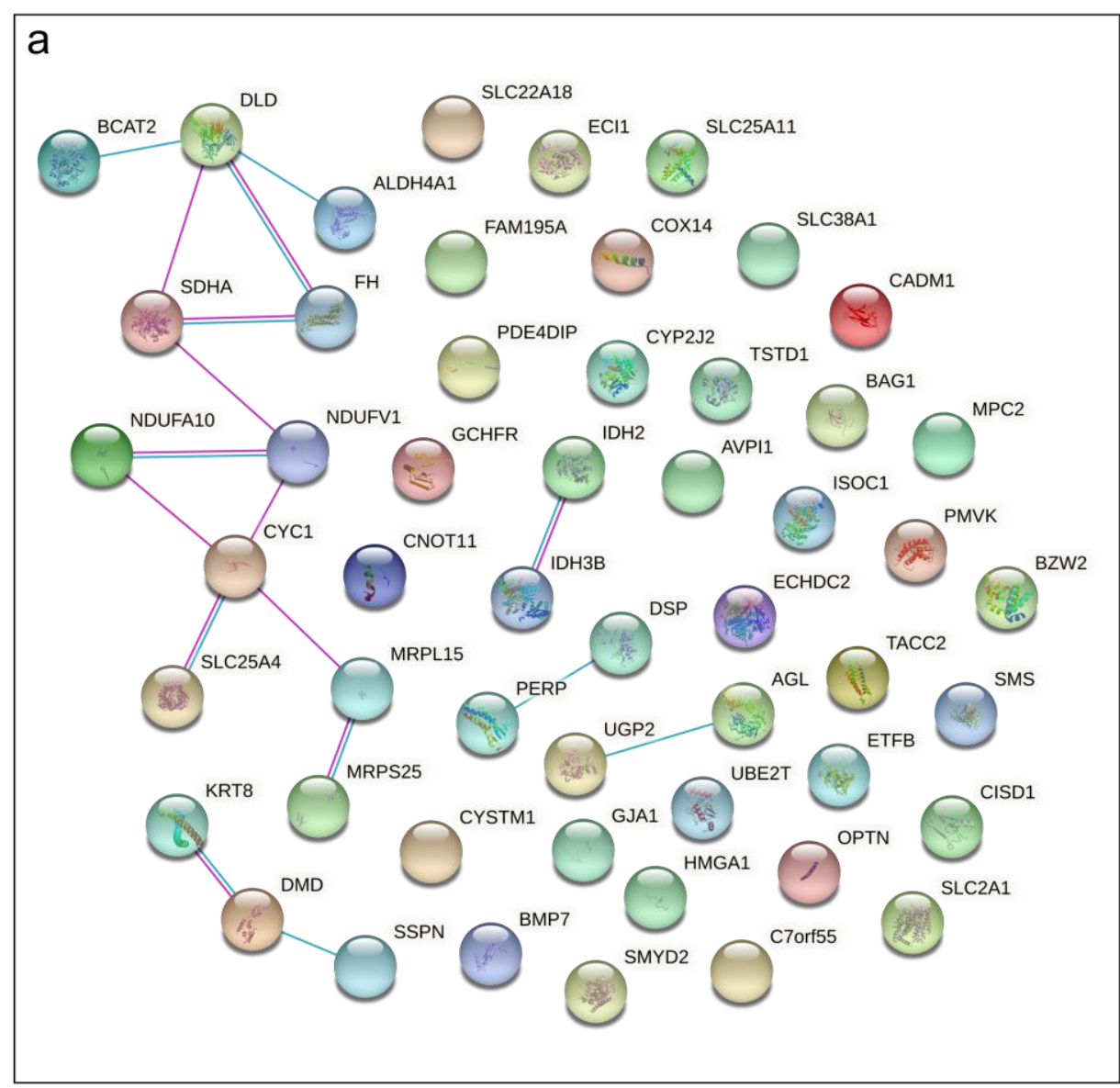

\begin{tabular}{|l|l|c|c|l|}
\hline \multicolumn{1}{|c|}{ Name } & \multicolumn{1}{|c|}{ Source } & P Value & FDR B\&H & \multicolumn{1}{|c|}{ Genes } \\
\hline G0:0055114 & Embryonic Lethality During Organogenesis, complete penetrance & $3.46 E-10$ & $1.58 E-07$ & $\begin{array}{l}\text { CYC1, TSTD1, CYP212, CISD1, AGL, ETFB, UGP2, DLD, BCAT2, } \\
\text { ALDH4A1, IDH2, IDH3B, FH, ECI1, NDUFA10, ECHDC2, NDUFV1, } \\
\text { SDHA }\end{array}$ \\
\hline G0:0006091 & Generation of Precursor Metabolites and Energy & $8.31 E-11$ & $5.05 E-08$ & $\begin{array}{l}\text { CYC1, SLC25A4, CISD1, AGL, ETFB, UGP2, DLD, ALDH4A1, IDH2, } \\
\text { IDH3B, FH, NDUFA10, NDUFV1, SDHA, }\end{array}$ \\
\hline G0:0006811 & Ion Transport & $7.48 E-04$ & $2.08 E-02$ & $\begin{array}{l}\text { CYC1, SLC38A1, GJA1, SLC22A18, MPC2, SLC25A4, DLD, } \\
\text { SLC25A11, DMD, NDUFA10, SLC2A1, NDUFV1, SDHA }\end{array}$ \\
\hline
\end{tabular}

Figure 5. Comparison of commonly expressed genes between first trimester fetal heart cardiomyocytes and placenta cytotrophoblasts. a. Protein-protein interactions of the commonly expressed genes between heart cardiomyocytes and placenta cytotrophoblasts.

b. GoBiological processes enrichment analysis of commonly expressed genes between heart cardiomvocvtes and placenta cvtotrophoblasts.

\section{Conclusion}

To date, efforts to understand the mechanistic origins of congenital heart defects have largely ignored the impact of the placenta. Given the heart and the placenta develop concurrently in early 
gestation, and the direct physiological connection, there is the potential for investigations that ignore the role the placenta in the development of CHDs to miss crucial causative steps. Systematic analysis of single-cell gene expression profiles between first trimester heart and placenta cell types has revealed commonly expressed genes and biological pathways that are essential for normal cell and organ function and known to be associated with both CHDs and placenta-related obstetric diseases. Hence, providing further evidence that future research into the mechanisms behind CHD development need to acknowledge the contribution of the placenta.

\section{Methods}

\section{Data Preparation}

Single cell sequencing data was obtained from two publicly available repositories: Cui et al. GEO GSE106118 and Vento-Tormo et al. ebi ArrayExpress (E-MTAB-6701 and E-MTAB-6678). The Vento-Tormo data was downloaded as filtered, unnormalized counts. The Cui data was downloaded as already TPM-normalized. To account for the different scales of these data, both datasets were separately normalized with a natural log transformation, and then batch correction (canonical correlation analysis) was applied using the R package Seurat ${ }^{54}$.

\section{Shared genes between first trimester heart and placenta cells}

As a first step, the $R$ package Seurat was used to identify cell-specific differentially expressed genes in a tissue-specific manner. Using a Wilcoxon Rank Sum test and a one-versus-all design, the mean expression of a gene for one cell type was compared to the mean expression of all other cell types. To define cell-specific differential expression, stringent statistical thresholds were used to minimize the proportion of false positives: the minimum log-fold change was set to $>0.2$, Bonferroni-adjusted $p$-value of $<0.01$, and the difference in the percentage of cells showing expression of a gene between the cell type of interest and all other cell types was $>5 \%$. To find commonly expressed genes, the cell-specific differentially expressed genes were overlapped in a pairwise fashion between heart and placenta cell types.

\section{Analysis of shared genes between first trimester heart and placenta cells}

The commonly expressed genes between cell types of interest were entered into ToppFun (ToppGene Suite V25: ${ }^{55}$ ) for enrichment analysis of GO Biological Process, Mice Phenotypes and Human Diseases. P values were calculated using the Hypergeometric Probability Mass Function and false discovery rate corrected using Benjamini-Hochberg methods. Potential protein interactions of shared genes of interest between cell types were analyzed using STRING (Database $\mathrm{V} 11.0^{56}$ ). Only known protein-protein associations from curated databases and/or experimentally determined were assessed.

\section{References}

1 van der Linde, D. et al. Birth prevalence of congenital heart disease worldwide: a systematic review and meta-analysis. J Am Coll Cardiol 58, 2241-2247, doi:10.1016/j.jacc.2011.08.025 (2011).

2 Yang, Q. et al. Racial differences in infant mortality attributable to birth defects in the United States, 1989-2002. Birth Defects Res A Clin Mol Teratol 76, 706-713, doi:10.1002/bdra.20308 (2006).

3 Leirgul, E. et al. Birth prevalence of congenital heart defects in Norway 1994-2009--a nationwide study. Am Heart J 168, 956-964, doi:10.1016/j.ahj.2014.07.030 (2014). 
4 Tyser, R. C. et al. Calcium handling precedes cardiac differentiation to initiate the first heartbeat. Elife 5, doi:10.7554/eLife.17113 (2016).

5 Linask, K. K. The heart-placenta axis in the first month of pregnancy: induction and prevention of cardiovascular birth defects. J Pregnancy 2013, 320413, doi:10.1155/2013/320413 (2013). Khong, T. Y. Placental vascular development and neonatal outcome. Semin Neonatol 9, 255-263, doi:10.1016/j.siny.2003.11.010 (2004).

7 Kaufmann, P., Mayhew, T. M. \& Charnock-Jones, D. S. Aspects of human fetoplacental vasculogenesis and angiogenesis. II. Changes during normal pregnancy. Placenta 25, 114-126, doi:10.1016/j.placenta.2003.10.009 (2004).

8 Schleich, J. M. Images in cardiology. Development of the human heart: days 15-21. Heart 87, 487, doi:10.1136/heart.87.5.487 (2002).

9 Hemberger, M. \& Cross, J. C. Genes governing placental development. Trends Endocrinol Metab 12, 162-168, doi:10.1016/s1043-2760(01)00375-7 (2001).

10 Jarrell, D. K., Lennon, M. L. \& Jacot, J. G. Epigenetics and Mechanobiology in Heart Development and Congenital Heart Disease. Diseases 7, doi:10.3390/diseases7030052 (2019).

11 Huppertz, B. \& Peeters, L. L. Vascular biology in implantation and placentation. Angiogenesis 8, 157-167, doi:10.1007/s10456-005-9007-8 (2005).

Courtney, J. A., Cnota, J. F. \& Jones, H. N. The Role of Abnormal Placentation in Congenital Heart Disease; Cause, Correlate, or Consequence? Front Physiol 9, 1045, doi:10.3389/fphys.2018.01045 (2018).

13 Shen, H. et al. Extracardiac control of embryonic cardiomyocyte proliferation and ventricular wall expansion. Cardiovasc Res 105, 271-278, doi:10.1093/cvr/cvu269 (2015).

14 Maslen, C. L. Recent Advances in Placenta-Heart Interactions. Front Physiol 9, 735, doi:10.3389/fphys.2018.00735 (2018).

15 Zaidi, S. \& Brueckner, M. Genetics and Genomics of Congenital Heart Disease. Circ Res 120, 923940, doi:10.1161/CIRCRESAHA.116.309140 (2017).

16 Yuan, S., Zaidi, S. \& Brueckner, M. Congenital heart disease: emerging themes linking genetics and development. Curr Opin Genet Dev 23, 352-359, doi:10.1016/j.gde.2013.05.004 (2013).

17 Vento-Tormo, R. et al. Single-cell reconstruction of the early maternal-fetal interface in humans. Nature 563, 347-353, doi:10.1038/s41586-018-0698-6 (2018).

18 Cui, Y. et al. Single-Cell Transcriptome Analysis Maps the Developmental Track of the Human Heart. Cell Rep 26, 1934-1950 e1935, doi:10.1016/j.celrep.2019.01.079 (2019).

19 Harvey, R. P. Patterning the vertebrate heart. Nat Rev Genet 3, 544-556, doi:10.1038/nrg843 (2002).

20 von Gise, A. \& Pu, W. T. Endocardial and epicardial epithelial to mesenchymal transitions in heart development and disease. Circ Res 110, 1628-1645, doi:10.1161/CIRCRESAHA.111.259960 (2012).

21 Boss, A. L., Chamley, L. W. \& James, J. L. Placental formation in early pregnancy: how is the centre of the placenta made? Hum Reprod Update 24, 750-760, doi:10.1093/humupd/dmy030 (2018).

22 Burton, G. J., Charnock-Jones, D. S. \& Jauniaux, E. Regulation of vascular growth and function in the human placenta. Reproduction 138, 895-902, doi:10.1530/REP-09-0092 (2009).

23 Artavanis-Tsakonas, S., Rand, M. D. \& Lake, R. J. Notch signaling: cell fate control and signal integration in development. Science 284, 770-776 (1999).

24 Niessen, K. \& Karsan, A. Notch signaling in cardiac development. Circ Res 102, 1169-1181, doi:10.1161/CIRCRESAHA.108.174318 (2008).

25 Preuss, C. et al. Family Based Whole Exome Sequencing Reveals the Multifaceted Role of Notch Signaling in Congenital Heart Disease. PLoS Genet 12, e1006335, doi:10.1371/journal.pgen.1006335 (2016). 
Swiatek, P. J., Lindsell, C. E., del Amo, F. F., Weinmaster, G. \& Gridley, T. Notch1 is essential for postimplantation development in mice. Genes Dev 8, 707-719, doi:10.1101/gad.8.6.707 (1994).

27 Krebs, L. T. et al. Notch signaling is essential for vascular morphogenesis in mice. Genes Dev 14, 1343-1352 (2000).

28 Limbourg, F. P. et al. Essential role of endothelial Notch1 in angiogenesis. Circulation 111, 18261832, doi:10.1161/01.CIR.0000160870.93058.DD (2005).

29 Magness, R. R., Sullivan, J. A., Li, Y., Phernetton, T. M. \& Bird, I. M. Endothelial vasodilator production by uterine and systemic arteries. VI. Ovarian and pregnancy effects on eNOS and NO(x). Am J Physiol Heart Circ Physiol 280, H1692-1698, doi:10.1152/ajpheart.2001.280.4.H1692 (2001).

30 Hefler, L. A., Tempfer, C. B., Moreno, R. M., O'Brien, W. E. \& Gregg, A. R. Endothelial-derived nitric oxide and angiotensinogen: blood pressure and metabolism during mouse pregnancy. $\mathrm{Am} J$ Physiol Regul Integr Comp Physiol 280, R174-182, doi:10.1152/ajpregu.2001.280.1.R174 (2001).

31 Kusinski, L. C. et al. eNOS knockout mouse as a model of fetal growth restriction with an impaired uterine artery function and placental transport phenotype. Am J Physiol Regul Integr Comp Physiol 303, R86-93, doi:10.1152/ajpregu.00600.2011 (2012).

32 Kobashi, G. et al. Endothelial nitric oxide synthase gene (NOS3) variant and hypertension in pregnancy. Am J Med Genet 103, 241-244 (2001).

33 Feng, Q. et al. Development of heart failure and congenital septal defects in mice lacking endothelial nitric oxide synthase. Circulation 106, 873-879, doi:10.1161/01.cir.0000024114.82981.ea (2002).

34 Bloch, W. et al. Nitric oxide synthase expression and role during cardiomyogenesis. Cardiovasc Res 43, 675-684, doi:10.1016/s0008-6363(99)00160-1 (1999).

35 Zhou, K. et al. Genetic variants of the endothelial NO synthase gene (eNOS) may confer increased risk of sporadic congenital heart disease. Genet Mol Res 13, 3805-3811, doi:10.4238/2014.May.16.4 (2014).

36 Knöfler, M. \& Pollheimer, J. IFPA Award in Placentology lecture: molecular regulation of human trophoblast invasion. Placenta 33, S55-S62 (2012).

37 Kaufmann, P., Black, S. \& Huppertz, B. Endovascular trophoblast invasion: implications for the pathogenesis of intrauterine growth retardation and preeclampsia. Biology of reproduction 69, 17 (2003).

38 Cedergren, M. I. \& Kallen, B. A. Obstetric outcome of 6346 pregnancies with infants affected by congenital heart defects. Eur J Obstet Gynecol Reprod Biol 125, 211-216, doi:10.1016/j.ejogrb.2005.07.006 (2006).

39 Brodwall, K. et al. Possible Common Aetiology behind Maternal Preeclampsia and Congenital Heart Defects in the Child: a Cardiovascular Diseases in Norway Project Study. Paediatr Perinat Epidemiol 30, 76-85, doi:10.1111/ppe.12252 (2016).

40 Liu, J. et al. There is a Strong Association between Early Preeclampsia and Congenital Heart Defects: A Large Population-Based, Retrospective Study. Gynecol Obstet Invest, 1-8, doi:10.1159/000506804 (2020).

41 Boyd, H. A. et al. Association Between Fetal Congenital Heart Defects and Maternal Risk of Hypertensive Disorders of Pregnancy in the Same Pregnancy and Across Pregnancies. Circulation 136, 39-48, doi:10.1161/CIRCULATIONAHA.116.024600 (2017).

42 Auger, N., Fraser, W. D., Healy-Profitos, J. \& Arbour, L. Association Between Preeclampsia and Congenital Heart Defects. JAMA 314, 1588-1598, doi:10.1001/jama.2015.12505 (2015).

43 Lash, G. E. et al. Regulation of extravillous trophoblast invasion by uterine natural killer cells is dependent on gestational age. Human reproduction 25, 1137-1145 (2010). 
44 Tantbirojn, P., Crum, C. \& Parast, M. Pathophysiology of placenta creta: the role of decidua and extravillous trophoblast. Placenta 29, 639-645 (2008).

45 Hove, J. R. et al. Intracardiac fluid forces are an essential epigenetic factor for embryonic cardiogenesis. Nature 421, 172-177, doi:10.1038/nature01282 (2003).

46 Goenezen, S., Rennie, M. Y. \& Rugonyi, S. Biomechanics of early cardiac development. Biomech Model Mechanobiol 11, 1187-1204, doi:10.1007/s10237-012-0414-7 (2012).

47 Midgett, M., Thornburg, K. \& Rugonyi, S. Blood flow patterns underlie developmental heart defects. Am J Physiol Heart Circ Physiol 312, H632-H642, doi:10.1152/ajpheart.00641.2016 (2017).

48 Guo, Y. \& Pu, W. T. Cardiomyocyte Maturation: New Phase in Development. Circ Res 126, 10861106, doi:10.1161/CIRCRESAHA.119.315862 (2020).

49 Jopling, C. et al. Zebrafish heart regeneration occurs by cardiomyocyte dedifferentiation and proliferation. Nature 464, 606-609, doi:10.1038/nature08899 (2010).

50 Porrello, E. R. et al. Transient regenerative potential of the neonatal mouse heart. Science 331, 1078-1080, doi:10.1126/science.1200708 (2011).

51 Aplin, J. D. et al. Trophoblast differentiation: progenitor cells, fusion and migration -- a workshop report. Placenta 27 Suppl A, S141-143, doi:10.1016/j.placenta.2006.01.011 (2006).

52 Talman, V. \& Ruskoaho, H. Cardiac fibrosis in myocardial infarction-from repair and remodeling to regeneration. Cell Tissue Res 365, 563-581, doi:10.1007/s00441-016-2431-9 (2016).

53 Longtine, M. S., Chen, B., Odibo, A. O., Zhong, Y. \& Nelson, D. M. Villous trophoblast apoptosis is elevated and restricted to cytotrophoblasts in pregnancies complicated by preeclampsia, IUGR, or preeclampsia with IUGR. Placenta 33, 352-359, doi:10.1016/j.placenta.2012.01.017 (2012).

54 Zhang, F., Wu, Y. \& Tian, W. A novel approach to remove the batch effect of single-cell data. Cell discovery 5, 1-4 (2019).

55 Chen, J., Bardes, E. E., Aronow, B. J. \& Jegga, A. G. ToppGene Suite for gene list enrichment analysis and candidate gene prioritization. Nucleic Acids Res 37, W305-311, doi:10.1093/nar/gkp427 (2009).

56 Szklarczyk, D. et al. STRING v11: protein-protein association networks with increased coverage, supporting functional discovery in genome-wide experimental datasets. Nucleic Acids Res 47, D607-D613, doi:10.1093/nar/gky1131 (2019).

Acknowledgments: This study was funded by National Institutes Health award R01HD091527 (HNJ).

Author contributions: RLW designed the analyses, interpreted the data and wrote and drafted the manuscript. VY analyzed and interpreted the data and edited the manuscript. JC designed the analyses and edited the manuscript. AT interpreted the data and edited the manuscript. JC conceived the work and edited the manuscript. HNJ conceived the work, designed the analyses, obtained the funding and edited the manuscript. All authors have reviewed and approved the submitted version.

Competing Interests: the authors declare no conflicts of interest

Data Availability: Single cell sequencing data can be obtained from two publicly available repositories: GEO (GSE106118) and ebi ArrayExpress (E-MTAB-6701 and E-MTAB-6678). 